\title{
Improving the Resistance to Humid Heat Sterilization of EVOH Copolymers Through Blending
}

\author{
Amparo Lopez-Rubio, ${ }^{*}$ Jose M. Lagaron \\ Novel Materials and Nanotechnology Lab., Institute of Agrochemistry and Food Technology (IATA), CSIC, \\ 46100 Burjassot, Spain
}

Received 22 May 2007; accepted 26 December 2007

DOI 10.1002/app.27991

Published online 20 March 2008 in Wiley InterScience (www.interscience.wiley.com).

\begin{abstract}
The effect of retorting, i.e., combined temperature and high relative humidity conditions, on the morphology, structure, and thermal characteristics of a number of extruded films containing binary and ternary blends of a $32 \mathrm{~mol} \%$ ethylene-vinyl alcohol copolymer $(\mathrm{EVOH})$ with amorphous polyamide (aPA) and nylon-containing ionomer as blending additives was carried out. Each film was investigated by differential scanning calorimetry, attenuated total reflectance Fourier transformed infrared spectroscopy (ATR-FTIR), scanning electron microscopy (SEM), and synchrotron X-ray scattering. The oxygen transmission rate of multilayer structures having as barrier element the cited blends was also measured and the results were interpreted in terms of the blends' particular structural and morphological features. From the results
\end{abstract}

it was found that the thermal properties, the crystalline structure, and the water resistance of the blends were improved upon retorting compared with neat EVOH. However, only the binary blend with aPA showed a real enhancement in the oxygen barrier properties immediately after retorting compared with neat EVOH. This unprecedented and surprising effect was additionally ascribed to the retorting-induced compatibilization between $\mathrm{EVOH}$ and aPA components of the blend as determined by SEM. (C) 2008 Wiley Periodicals, Inc. J Appl Polym Sci 109: 174181,2008

Key words: ethylene vinyl-alcohol copolymers; amorphous polyamide; nylon-containing ionomer; packaged food retorting; barrier properties

\section{INTRODUCTION}

Ethylene-vinyl alcohol (EVOH) random copolymers are a family of semicrystalline materials with excellent barrier properties to gases and hydrocarbons along with improved chemical resistance. These materials are being increasingly used in the food packaging industry as barrier layers to minimize the ingress of oxygen and the loss of flavors, which increases the packaged product's shelf-life. The main drawback of these copolymers is their high hydrophilicity, which most often requires them to be used as intermediate layer in multilayer polymeric structures protected by hydrophobic materials such as polyolefins. ${ }^{1-3}$

Many oxygen-sensitive food products undergo thermal processes such as hot filling or sterilization (retorting) inside plastic packages. Therefore, it is a

*Present address: Bragg Institute, Australian Nuclear Science and Technology Organisation, PMB 1, Menai, NSW 2234, Australia.

Correspondence to: J. M. Lagaron (lagaron@iata.csic.es).

Contract grant sponsor: European Commission; contract grant number: HPRI-CT-1999-00040/2001-00140.

Contract grant sponsor: The Nippon Synthetic Chemical Industry Co. Ltd. (NIPPON GOHSEI), Japan.

Journal of Applied Polymer Science, Vol. 109, 174-181 (2008)

(C) 2008 Wiley Periodicals, Inc.

\section{(\$ILEY}

InterScience general requirement for the selected packaging system to have a high oxygen barrier and resistance to wet and humid thermal treatments. Sterilization processes that use heated water vapor as the heat transfer medium (retorting treatment) can potentially alter the packaging structure compromising the barrier properties, and shelf-life of the packaged product. ${ }^{2,3}$

Previous works on multilayer structures containing $\mathrm{EVOH}$ copolymers as the high barrier element indicated that, during standard industrial food packaging sterilization processes, some of the pressurized water vapor was capable of traversing the external hydrophobic layers made of polypropylene and sorbed into the EVOH intermediate layer. Consequently, the oxygen permeability of this high-barrier material did increase by up to three orders of magnitude depending on grade, leading to a long-standing decrease in barrier properties, and hence, compromising packaged food quality and safety. ${ }^{1}$

Among the strategies used to diminish the barrier deterioration of $\mathrm{EVOH}$ when exposed to high humidity conditions, the use of blends has been widely considered. ${ }^{4-7}$ The main drawback for blending $\mathrm{EVOH}$ with other polymers is the strong polymer self-association promoted by the hydroxyl groups, which leads to poor compatibility.

Binary and ternary blends of amorphous polyamide (aPA) and nylon-containing ionomer with $\mathrm{EVOH}$ 
have proven to have beneficial effects such as improved processability during thermoforming. Even when these blends were found to be immiscible, a good phase dispersion and adhesion at the interphase were generally found. ${ }^{5,6}$ From a barrier perspective, the materials were found to provide a positive deviation from the Maxwell model in oxygen permeability, which led to better barrier properties than expected. ${ }^{6}$ Furthermore, both the aPA and the nylon-containing ionomer exhibited lower water sorption than the hydrophilic EVOH.

In this study, it is reported for the first time on the effect of retorting on two binary blends and one ternary blend of EVOH with aPA and of a nylon-containing ionomer in terms of structural, thermal, and oxygen barrier characteristics. The results are also compared with those obtained for pure EVOH.

\section{EXPERIMENTAL}

\section{Materials and retorting treatment}

Two binary blends and a ternary blend of commercially available EVOH copolymer (EVOH with $32 \mathrm{~mol} \%$ of ethylene, Soarnol ${ }^{\circledR}$ DC3202) supplied by The Nippon Synthetic Chemical Industry Co. (NIPPON GOHSEI, Japan), aPA (Selar ${ }^{\circledR}$ aPA UX2034, Wilmington, DE), polymerized by condensation of hexamethylene diamine and a mixture of 70/ 30 isophthalic and terephthalic acids), and a nyloncontaining ionomer (Surlyn ${ }^{\circledR}$ AM-7938) from Dupont de Nemours were prepared by blending in an internal mixer at $230^{\circ} \mathrm{C}$ for $5 \mathrm{~min}$. Polymer films $(\sim 45$ $\mu \mathrm{m}$ thick) were obtained by cast extrusion of the grinded blends. The compositions in weight of the blends used in this study were $80 / 20,80 / 20$, and $80 / 10 / 10$ for the $\mathrm{EVOH} / \mathrm{aPA}, \mathrm{EVOH} /$ Ionomer, and EVOH/aPA/Ionomer, respectively. The samples tested for oxygen permeability were vacuum thermosealed between polypropylene (PP) layers $(\sim 70$ $\mu \mathrm{m}$ thick) prior to the thermal treatment.

The different materials, with and without PP protection, were retorted in a sterilization autoclave using industrial standard conditions at $121^{\circ} \mathrm{C}$ for $20 \mathrm{~min}$ and were subsequently vapor purged, removed from the autoclave, and allowed to cool down at room temperature.

\section{Methods}

After the retorting treatment, the multilayer structures were delaminated and the intermediate barrier layer was immediately placed in the permeability cell and its oxygen transmission rate $\left(\mathrm{O}_{2} \mathrm{TR}\right)$ was measured as a function of time. $\mathrm{O}_{2} \mathrm{TR}$ values were obtained with an OXTRAN 2/20 (Mocon Inc., Minneapolis, MN). The samples were placed in a $5-\mathrm{cm}^{2}$ test cell and the measurements were made using a gas flux of $10 \mathrm{~mL} / \mathrm{min}$, at $24^{\circ} \mathrm{C}$ and a relative humidity of $0 \%$. The $\mathrm{O}_{2} \mathrm{TR}$ values were corrected for the thickness of the films and for the differential pressure gradient, i.e., 1 atm. The samples were tested in duplicate and the lowest permeability value is reported as is customary in barrier testing. Nevertheless, different specimens of the various samples provided fairly similar results with variability lower than $4 \%$ given the good quality of the extruded films generated.

Attenuated total reflection (ATR) infrared (IR) experiments were carried out coupling the Golden Gate ATR accessory (Specac, Orpiugtou, UK) to a Fourier transformed infrared (FTIR) Tensor 37 (Bruker, Rheiustetlen, Germany) spectrometer with a resolution of $4 \mathrm{~cm}^{-1}$. Typical acquisition times were about $10 \mathrm{~s}$.

Differential scanning calorimetry (DSC) experiments were carried out in a Perkin-Elmer DSC-7 calorimeter. The heating and cooling rate for the runs was $10^{\circ} \mathrm{C} / \mathrm{min}$, with the typical sample weight around $4 \mathrm{mg}$. The temperature range of the assays was from 50 to $250^{\circ} \mathrm{C}$. The thermal runs of the samples were subtracted from the thermograms of an empty pan to enhance accuracy and repeatability, and calibration was performed using an indium sample. All tests were carried out in duplicate to check for repeatability and the mean values and standard errors are provided.

Wide angle X-ray scattering (WAXS) experiments were carried out at the synchrotron radiation source in the soft condensed matter beam at HASYLAB (DESY) in Hamburg (Germany). Scattering patterns were recorded using a one-dimensional detector and an incident radiation wavelength, $\lambda$, of $0.15 \mathrm{~nm}$. WAXS data were corrected for detector response and beam intensity and calibrated against a PET standard. ${ }^{8}$

For scanning electron microscopy (SEM) observation, the samples were fractured in liquid nitrogen and mounted on a sample holder. The fracture surface of the different samples was sputtered with $\mathrm{Au} / \mathrm{Pd}$ in a vacuum. The SEM microphotographs (S4100, Hitachi, Osaka, Japan) were taken with an accelerating voltage of $10 \mathrm{keV}$ on the sample thickness.

\section{RESULTS AND DISCUSSION}

When conventional grades of EVOH are subjected to a retorting experiment in an autoclave without the hydrophobic protection of, for instance, some polyolefinic layers, the harmful effects of this treatment over the structure of the polymer becomes noticeable to the naked eye. The specimens suffered, immediately after retorting, from dimensional stability 

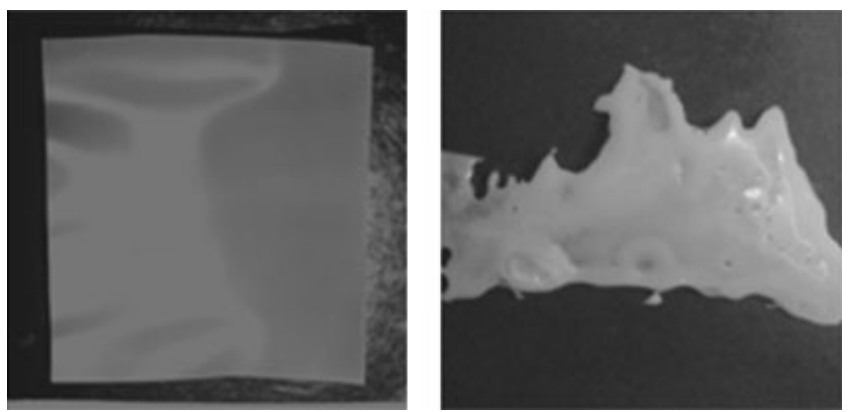

Figure 1 EVOH32 before (left) and after (right) retorting in autoclave $\left(121^{\circ} \mathrm{C}, 20 \mathrm{~min}\right)$.

losses, underwent whitening and loss of transparency, and became strongly plasticized, i.e., rubberylike (see Fig. 1).

Prior to a more exhaustive analytical characterization of the effects of retorting on the materials, photographs were also taken on the EVOH barrier blends. The aim of the visual characterization was to check whether the dimensional integrity and appearance were also detrimentally affected by the treatment.

Figure 2 indicates that the EVOH blends with aPA are not transparent but opaque, which could, in principle, limit the application of these materials in certain food packaging applications where transparency is required. This figure also shows that although in the retorted specimen the loss of integrity is less pronounced than in $\mathrm{EVOH}$ alone, the blend sample also shows damage and extensive plasticization, which is not surprising given the hydrophilic character of both materials.

However, for the case of the EVOH32/ionomer, Figure 3 indicates that the shape and form of the sheet is largely maintained, although voids and bubbles are still present in the retorted specimen. The ionomer polymer has much lower water uptake ${ }^{7}$ and sensitivity than the other two materials despite the fact that it contains a minor polyamide 6 fraction for compatibility with EVOH. This material is also characterized by the presence of ionic groups randomly
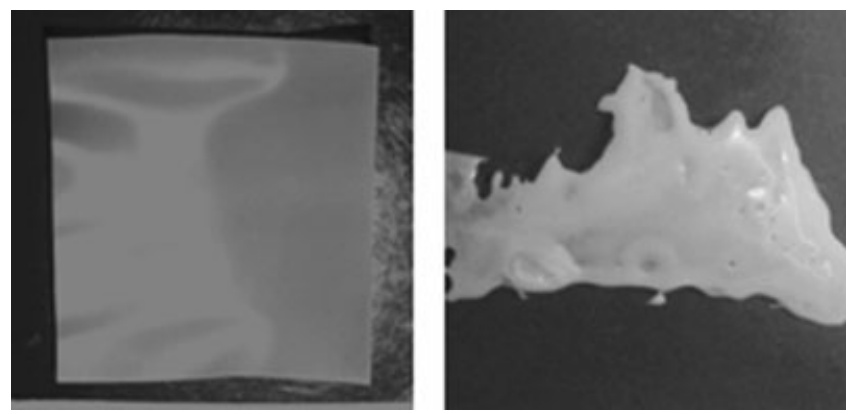

Figure 2 EVOH32/aPA blend before (left) and after (right) retorting in autoclave.
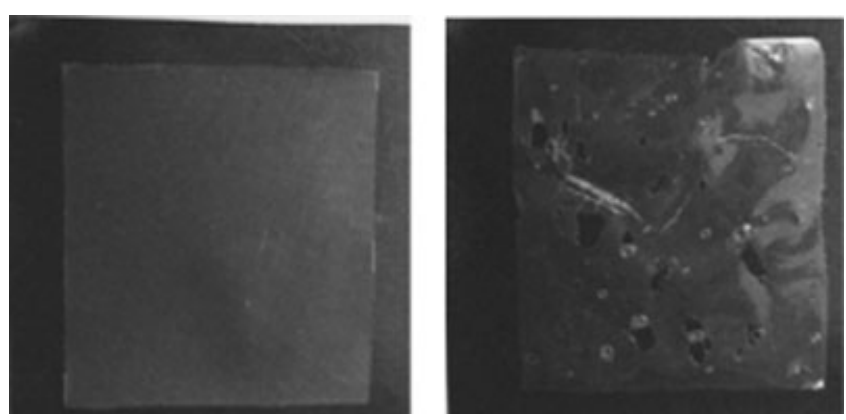

Figure 3 EVOH32/ionomer blend before (left) and after (right) retorting in autoclave.

dispersed across the polymer structure. However, as the temperature increases, the attractions between ionic groups decrease and the polymeric chains are allowed to freely move. The melting point of this material was found to be around $95^{\circ} \mathrm{C}$ in accordance with previous work, ${ }^{5}$ which means that during the retorting treatment in the autoclave at $121^{\circ} \mathrm{C}$, the ionomer fraction in the blend could in principle melt away. As this fraction softens and melts, the blend becomes ductile in some areas because of ingress of water vapor, and upon recrystallization this could lead to the observed voids. Curiously, the sample appears more transparent after retorting, suggesting morphological changes and finer phase dispersion.

The ternary blend, in comparison to neat $\mathrm{EVOH}$, also retained relatively well the dimensional stability (see Fig. 4), but the presence of aPA in the blend, apart from giving the whitish color to the blend, made the specimen also more water sensitive. Consequently, the sample shows more structural damage after the retorting treatment than the previous binary blend.

\section{Thermal characterization}

Previous characterization work in similar blends by DSC, WAXS, DMA, SEM, microhardness, and tensile testing indicated that the miscibility of the high-barrier EVOH32 copolymer with the aPA is very low-
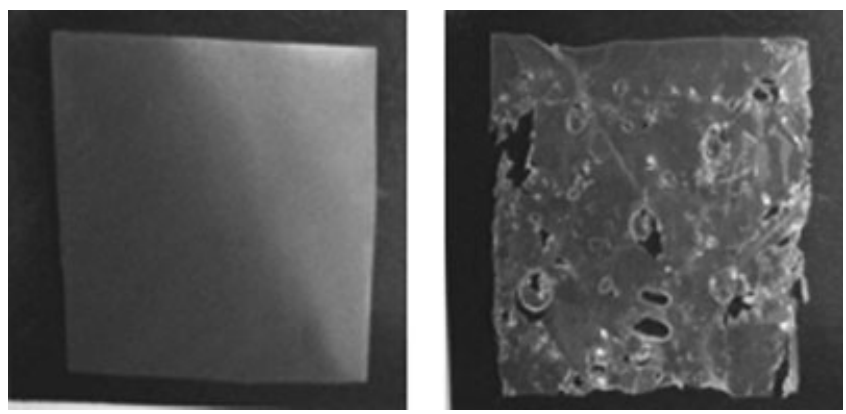

Figure 4 EVOH32/aPA/ionomer blend before (left) and after (right) retorting in autoclave. 
TABLE I

Mean Melting Point $\left(T_{m}\right)$, Melting Enthalpy $\left(\Delta H_{m}\right)$, Peak Width Values, and Corresponding Standard Deviation Data Measured by DSC During the First Heating Scan of the Different Samples Before and After Retorting

\begin{tabular}{lccc}
\hline & $T_{m}\left({ }^{\circ} \mathrm{C}\right)$ & $\Delta H_{m}(\mathrm{~J} / \mathrm{g})$ & Peak width $\left({ }^{\circ} \mathrm{C}\right)$ \\
\hline EVOH & & & \\
$\quad$ Untreated & $184.2 \pm 0.1$ & $83.5 \pm 3.2$ & $13.6 \pm 0.1$ \\
$\quad$ Retorted & $183.3 \pm 0.2$ & $82.2 \pm 2.6$ & $22.0 \pm 0.4$ \\
EVOH/aPA/Ionomer 80/10/10 & & & \\
$\quad$ Untreated & $179.9 \pm 0.2$ & $75.9 \pm 3.6$ & $16.3 \pm 0.1$ \\
$\quad$ Retorted & $180.2 \pm 0.2$ & $90.8 \pm 4.2$ & $13.6 \pm 0.2$ \\
EVOH/aPA 80/20 & & & \\
$\quad$ Untreated & $181.9 \pm 0.2$ & $61.5 \pm 2.1$ & $9.3 \pm 0.2$ \\
$\quad$ Retorted & $182.4 \pm 0.3$ & $75.9 \pm 3.3$ & $9.3 \pm 0.3$ \\
$\quad$ EVH/Ionomer 80/20 & & \\
$\quad$ Untreated & $179.9 \pm 0.2$ & $79.5 \pm 4.2$ & $16.3 \pm 0.1$ \\
$\quad$ Retorted & $181.2 \pm 0.3$ & $87.9 \pm 3.0$ & $12.8 \pm 0.2$ \\
\hline
\end{tabular}

Data on pure EVOH32 are included for comparison purposes.

hence the strong opacity and whitish color of the film-and clear phase segregation throughout composition was shown by DSC, DMA, and SEM. ${ }^{5,7}$ A two-phase structure was also observed in the $\mathrm{EVOH} /$ ionomer blends but, from the results, a better phase compatibility was inferred. This compatibility was thought to be enhanced by the presence of crystalline Nylon in the formulation of the ionomer. The lack of miscibility of EVOH with other materials whether of polar or apolar nature is related to the strong polymer self-association. As previously observed in other studies, ${ }^{2,3,9}$ as a consequence of retorting, this strong molecular interaction is greatly reduced. Water molecules from this preservation process intercept inter and intramolecular hydrogen bonding, leading to a strong deterioration in the crystalline morphology of the material. ${ }^{1,10}$ It is, therefore, of great interest to ascertain what structural changes underlay the morphological observations pictured above.

Table I displays the DSC parameters of the three blends before and after autoclaving. All the specimens were dried in a vacuum oven before the DSC measurements were taken to eliminate sorbed water. This also enables the estimation of enthalpies of the materials. The first heating run (from 50 to $250^{\circ} \mathrm{C}$ ) of the DSC experiments shows that, in agreement with previous results, ${ }^{5}$ the melting point of the blends were found to be somewhat lower than that of pure $\mathrm{EVOH} 32$. But, in contrast with the detrimental effects observed upon retorting of neat $\mathrm{EVOH}$ for the crystalline phase, the blends display similar or slightly higher melting point for the EVOH fraction than the untreated specimens suggesting more stable crystalline morphologies after retorting. Moreover, an increase in the melting enthalpy of the $\mathrm{EVOH}$ fraction was seen for every blend, which could be correlated with crystal growth and/or the formation of crystals during the retorting treatment. Thus, the presence of less hydrophilic phases in the blends reduces the damaging effect that the pressurized water vapor exerts over the $\mathrm{EVOH}$ phase. In fact, retorting results in a rather singular positive annealing effect for the crystallinity.

For the EVOH blends containing ionomer, a decrease in peak broadness after autoclaving of $\sim 17 \%$ and $\sim 21 \%$ for the ternary and binary blends, respectively, is also noticeable, which reflects a more homogeneous crystalline structure. However, the peak width of the EVOH/aPA blend did remain unmodified after the treatment. A reference to the latter observation will be made later in the article.

As discussed earlier, these results for the blends contrast with those previously obtained for the pure EVOH copolymer, in which the detrimental effects of this combined temperature and humidity treatment resulted in slight decreases in melting point and melting enthalpy and a significant increase in peak broadness.

\section{X-ray diffraction}

In Figures 5-7 the WAXS diffraction patterns of the dry and retorted blends are displayed.

The presence of ionomer both in the binary and in the ternary blends seems to have a beneficial effect on the retorted samples from a crystallinity viewpoint as discussed earlier. The diffraction peaks are narrower after the retorting treatment (see Figs. 5 and 6), indicating that very likely the crystalline structure is more robust and with larger crystal sizes in agreement with the trend shown by the DSC results.

On the other hand, the diffraction pattern of the binary blend $\mathrm{EVOH} / \mathrm{aPA}$ after retorting is broader than the untreated specimen. Broadening of a 


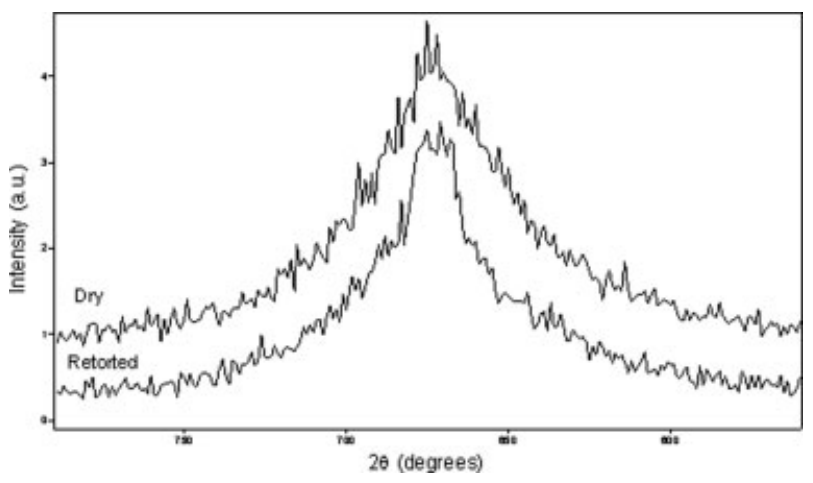

Figure 5 WAXS diffraction patterns of the binary blend EVOH/Ionomer 80/20 dry and retorted.

diffraction peak can usually be related to crystal size reduction (Scherrer formula) and/or to lattice distortions if we assume no instrumental effects. It is worth noting that the melting peak was not observed to narrow for this blend by DSC as for the others. However, because the retorted blend shows a trend for the melting peak to remain unmodified or to increase slightly by DSC, the peak broadening of the diffraction peak could preferentially be related to crystal heterogeneity because of lattice distortions and/or to an additional effect (see later in the text).

In a previous study, ${ }^{11}$ it was observed that the aPA develops lateral order in the form of crystals as a result of retorting. It is, thus, feasible that when the blend with $\mathrm{EVOH}$ is retorted, the aPA fraction also crystallizes to some extent giving rise to an overall peak broadening in the diffraction patterns of Figure 7. The previous study also showed that the formed aPA crystals are rather imperfect and heterogeneous in size. Crystallization of the aPA in the blend should generate a new endothermic peak in the first DSC run at around $190^{\circ} \mathrm{C}^{11}$ Nevertheless, it is feasible that the small amount of crystals formed within the $20 \mathrm{wt} \%$ fraction of aPA yield very small endotherms or are partially overlapped and, as a result, hidden under the melting endotherm of the EVOH matrix.

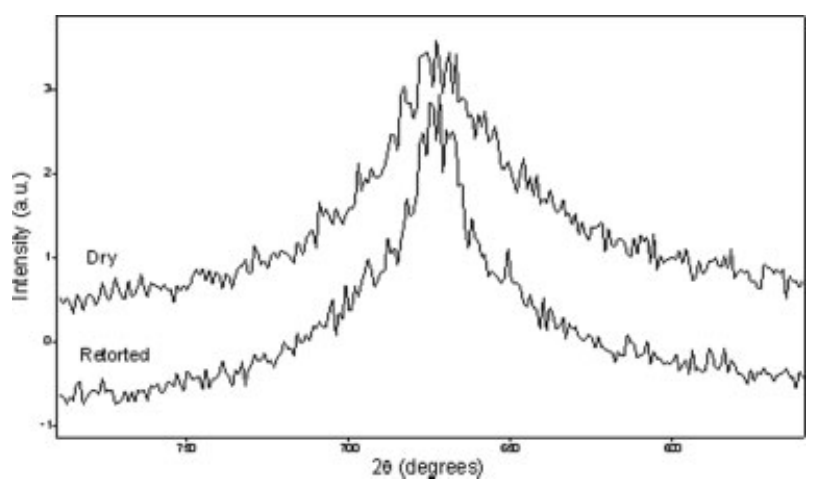

Figure 6 WAXS diffraction patterns of the ternary blend EVOH/aPA/Ionomer 80/10/10 dry and retorted.

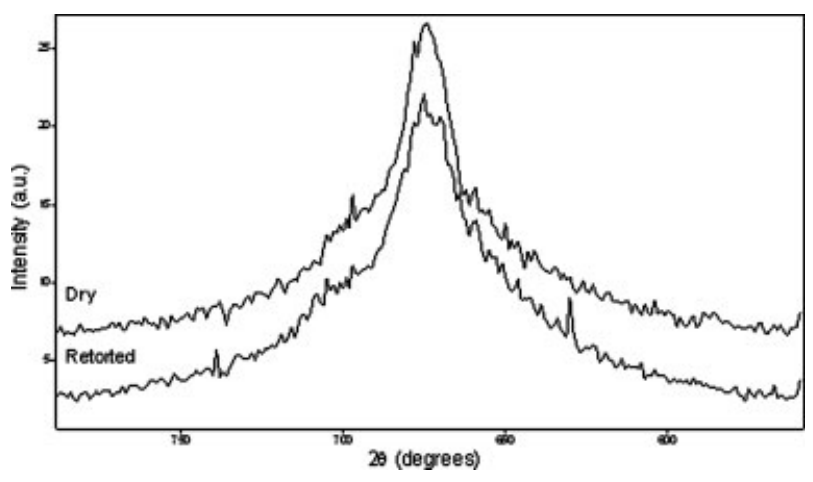

Figure 7 WAXS diffraction patterns of the binary blend EVOH/aPA 80/20 dry and retorted.

This effect could also account for the broadening of the diffraction peak discussed earlier. Another hypothesis that could be considered is the potential compatibilization of $\mathrm{EVOH}$ and aPA fractions induced by retorting and the subsequent partial cocrystallization of both polymer segments. Because the melting point of the aPA is slightly higher than that of EVOH but very close to it, water-induced cocrystallization may also explain the slight increase in the melting point of this blend or the particular broadening behavior of the diffraction peak. This will be further discussed in light of the FTIR results.

\section{FTIR spectroscopy}

Further analysis of the morphological changes undergone by the EVOH-based blends was also carried out by ATR-FTIR spectroscopy. Being aware of the strong infrared signal of water, the blend specimens were analyzed immediately after autoclaving, and again after being dried overnight in a vacuum oven.

Figure 8(a) shows the reference spectra of the dry and retorted $\mathrm{EVOH}$ and aPA pure materials, and Figure $8(\mathrm{~b})$ shows the spectra of dry, retorted, and retorted and dry specimens of the $\mathrm{EVOH} / \mathrm{aPA}$ blend. The most significant changes observed in $\mathrm{EVOH}$ after autoclaving are, first of all, the presence of sorbed water reflected by an increase in the $1658 \mathrm{~cm}^{-1}$ in-plane $\mathrm{OH}$ bending water band, and the decrease in the "crystallinity band" of EVOH at $1140 \mathrm{~cm}^{-1}$ [see arrows in Fig. 8(a)]. ${ }^{10}$ Regarding the pure aPA material, the molecular order developed upon retorting was evidenced by changes in several absorption bands ${ }^{11}$ (changes in shape, position, and relative intensity). Specifically, the amide I and II bands at 1630 and $1535 \mathrm{~cm}^{-1}$, respectively, which are considered to be conformationally sensitive, become narrower and are displaced toward lower wavenumbers, suggesting an increase in inter or intramolecular interactions and in molecular order. 
a)
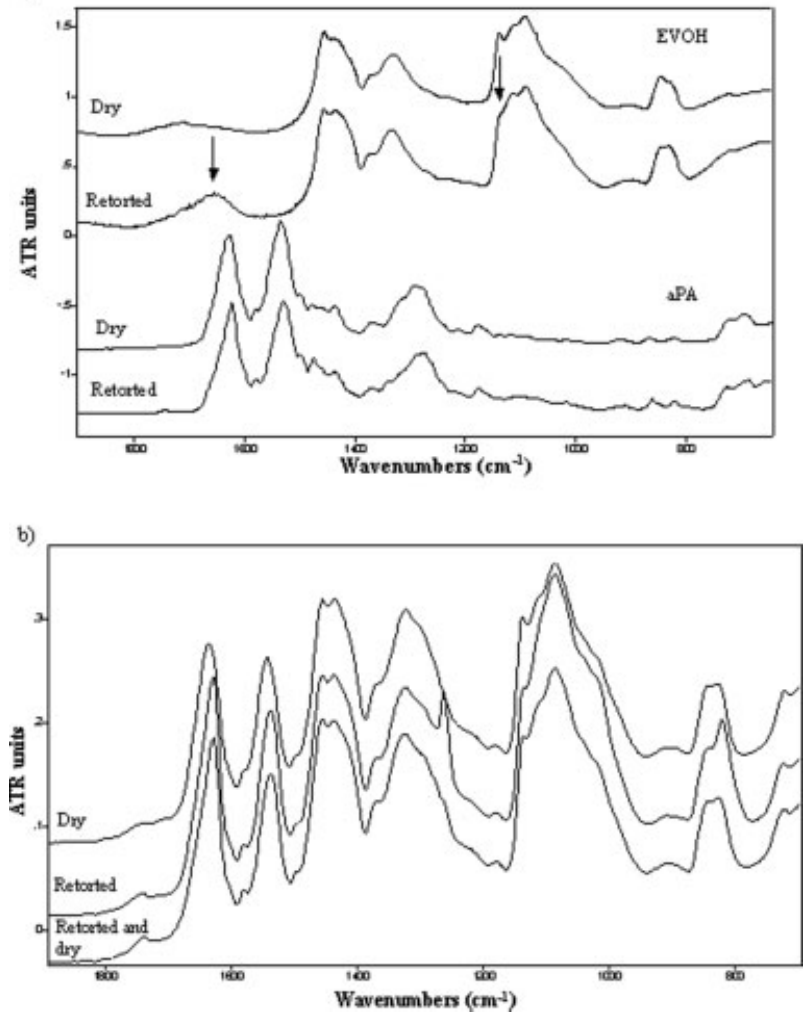

Figure 8 ATR-FTIR spectra of (a) EVOH32 dry and retorted and aPA dry and retorted and (b) dry, retorted, and retorted and dry binary blend EVOH/aPA 80/20.

In the spectra from the EVOH/aPA blend [Fig. 8(b)] it is observed that, in the autoclaved specimens, the amide I and II bands are also narrower and shifted. So, it is possible that the combined temperature and pressurized water vapor treatment induces, as suggested earlier, molecular order in the aPA fraction of the polymeric blend. Furthermore, in the just retorted sample, new bands arise at $\sim 1263$ and $822 \mathrm{~cm}^{-1}$. These bands were neither present in the dry neat polymer nor in the retorted one. Although, at the light of the DSC and WAXS results it was discussed the possibility of cocrystallization, the FTIR results suggest that this may not be feasible because bands from the individual crystalline phases of the two polymers are observed. The presence of new bands may arise from molecular "environment" and electronic perturbations, ${ }^{12}$ indicating that conformational changes may have occurred. Water is seen to have an important role in these changes because after drying the retorted sample these new bands disappear.

The increase in intensity of the bands adjacent to the "crystallinity" band of EVOH in the autoclaved blends makes difficult to draw a comparison in the EVOH crystallinity band. As mentioned earlier, after drying the autoclaved EVOH/aPA blend specimen, its spectrum is more similar to the spectrum of the untreated dry blend. However, even after drying, the amide I and II bands at 1630 and $1535 \mathrm{~cm}^{-1}$, respectively, remain displaced toward lower wavenumbers. These same spectral changes were previously observed for the retorted neat aPA material. This indicates that aPA is likely to rearrange and crystallize as a result of retorting, just, as it occurs in the neat material.

In Figure 9 the FTIR spectra of the dry, retorted, and retorted and dry ternary blend are displayed. From the spectrum of the dry specimen it could be inferred that the presence of both the aPA and the nylon-containing ionomer fractions restricts the ability of EVOH to crystallize (see "crystallinity band" at $1140 \mathrm{~cm}^{-1}$ ). This is in accordance with the lower melting point seen in the blend for the EVOH fraction. Nevertheless, after retorting, this band is seen to increase, suggesting that this humid heat treatment favors crystal development and homogeneity in the EVOH phase. Apart from the increase in the crystallinity of the EVOH fraction, the shifts of the amide I and II modes toward lower wavenumbers, suggesting again that aPA is also able to crystallize in the ternary blend as a consequence of autoclaving.

Substantial spectral changes are also observed between the dry and retorted binary EVOH/ionomer blend (see Fig. 10). Similarly as with the ternary blend, autoclaving of this sample leads to the development of crystallinity in the EVOH fraction, and the subsequent drying process further increases the intensity of the $1140 \mathrm{~cm}^{-1}$ crystallinity band. The $1500-1800 \mathrm{~cm}^{-1}$ range of the spectra is also greatly affected by retorting. The band at $1700 \mathrm{~cm}^{-1}$ completely and irreversibly disappears while there is a general increase in relative intensity within this spectral range. Again, water-induced molecular rearrangements must be behind these changes, but their precise ascription is unknown.

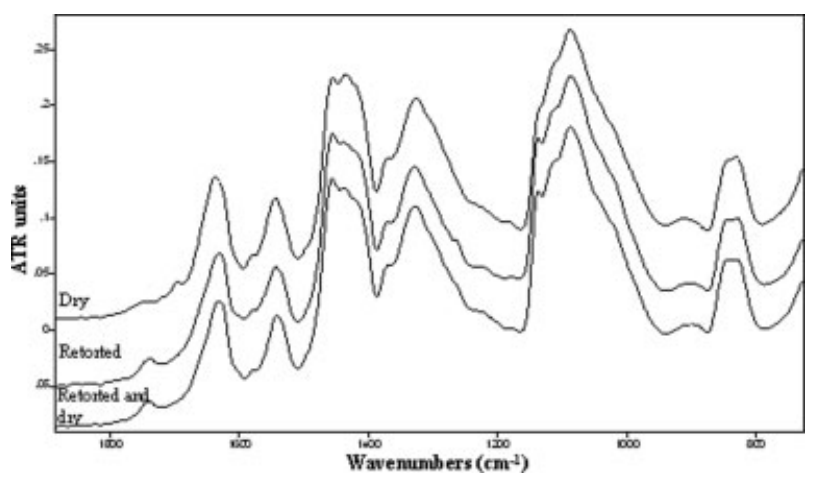

Figure 9 ATR-FTIR spectra of the ternary blend EVOH/ aPA/Ionomer 80/10/10: dry, retorted, and retorted and dry. 


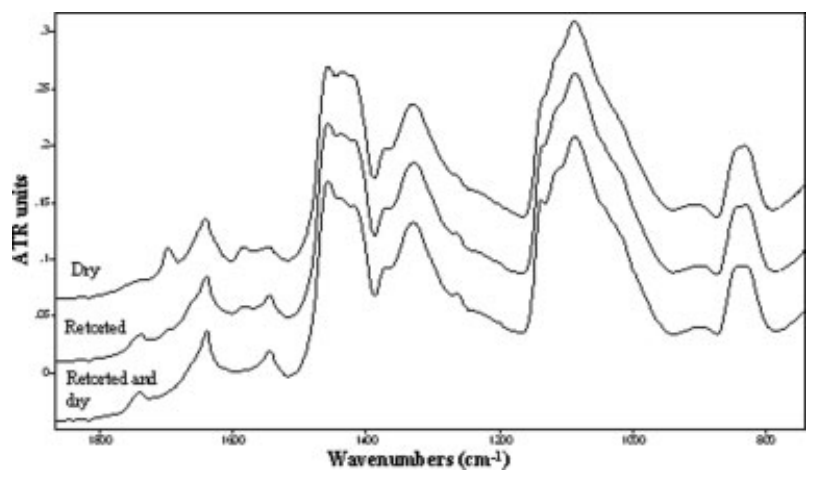

Figure 10 ATR-FTIR spectra of the binary blend EVOH/ Ionomer 80/20: dry, retorted, and retorted and dry.

\section{Oxygen permeability}

Figure 11 shows the oxygen permeability values measured at $0 \% \mathrm{RH}$ and $45^{\circ} \mathrm{C}$ of the binary and ternary blends before and after the retorting treatment. Because of the damage undergone by all samples upon direct retorting, the permeability to oxygen was measured in samples than underwent the autoclave process protected between PP layers. In this case, the effect of retorting leaves the samples much less with no apparent dimensional damage. Therefore, the barrier effect can be measured more consistently. Moreover, it is expected that this testing mode will resemble more closely the real application of the samples in retortable food packaging structures. In any case and from previous works in EVOH32, the plasticizing effect and the crystalline structural damage is still present in the barrier structure. ${ }^{1}$

From the permeability results it is observed that dry EVOH displays the lowest oxygen permeability, although the oxygen permeability of the dry blends is also very low (see Fig. 11). After retorting, every material undergoes an increase in permeability except the aPA. It has been recently found ${ }^{11}$ that,

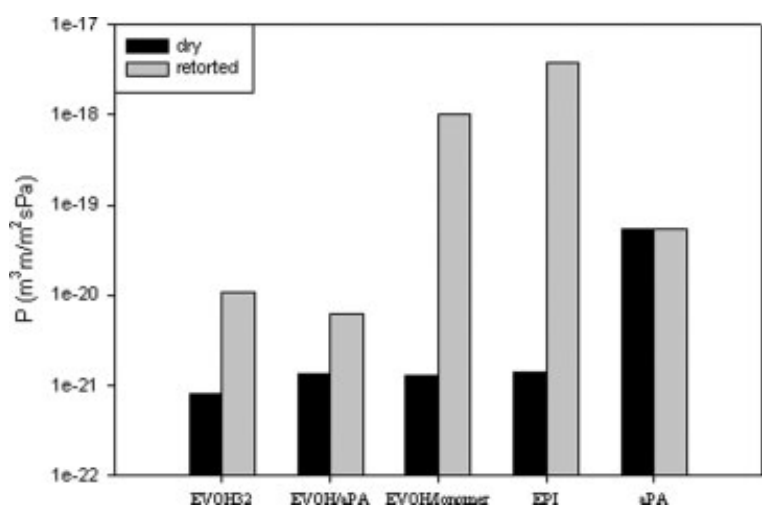

Figure 11 Oxygen permeability of pure components and blends measured at $0 \% \mathrm{RH}$ and $45^{\circ} \mathrm{C}$ before and after retorting. during retorting, this material is able to crystallize and this crystallization, together with the special interaction of this polymer with water, ${ }^{13,14}$ leads to a slight improvement in barrier properties. In the aPA the sorption of moisture does not disrupt the originally existing hydrogen bonding structure, but rather links to free amide moieties being the majority of the sorbed water in the form of clusters. ${ }^{13}$ There is a competing mechanism between oxygen and water clusters to fill the available free volume, which is favorable to water because of chemisorption. ${ }^{14}$ As a consequence, a decrease in the oxygen permeability is observed.

In addition to this and in accordance with all the above observations, retorting improves the compatibility of both materials as further inferred from the SEM images taken in the protected EVOH/aPA blend before and after retorting (see Fig. 12). This blend shows the best barrier performance immediately after retorting, suggesting that blending $\mathrm{EVOH}$ with aPA can reduce the detrimental effect of retorting in packaging structures. Figure 12 indicates that before retorting, and in agreement with previous results, ${ }^{5}$ the miscibility of high barrier EVOH and the amorphous PA is very poor and clear phase separation with some debonding is observed. However, the combined treatment of temperature and humidity allows for a better interaction between the two polymers and a more homogeneous microstructure is formed.

On the other hand, and in apparent contrast with the positive morphological findings reported earlier for the binary and ternary blends, the oxygen permeability of these blends containing ionomer greatly increased upon retorting, probably because of the softening and melting of the ionomer during the treatment leaving voids, i.e., free volume, in the structure (see Fig. 11). Furthermore, as these voids are kept in the blend structure, the original barrier properties of the materials were not seen to recover upon drying (results not shown).

As a result of all of the above, blends containing ionomer do not seem to be a good alternative to EVOH32 for food retorting applications. However,
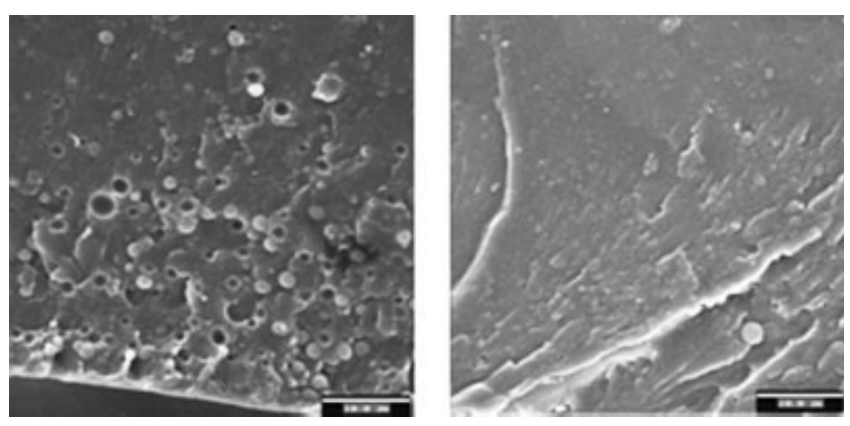

Figure 12 SEM images of the binary blend EVOH/aPA untreated (left) and retorted (right). Scale markers are $10 \mu \mathrm{m}$. 
blends of EVOH with aPA can have significant advantages after retorting because the barrier deterioration after retorting is clearly lower. Still, the lack of good optical properties of the latter blends could be a disadvantage in certain applications.

The authors thank The Nippon Synthetic Chemical Industry Co., Ltd. (NIPPON GOHSEI), Japan, for supplying EVOH samples. This work was performed at the synchrotron radiation facility in Hamburg (HASYLAB, DESY, Germany). The authors also thank Dr. S. S. Funari (A2 station) for experimental support.

\section{References}

1. Lopez-Rubio, A.; Hernandez-Muñoz, P.; Gimenez, E.; Yamamoto, T.; Gavara, R.; Lagaron, J. M. J Appl Polym Sci 2005, 96, 2192.

2. Lee, S. Y.; Kim, S. C. J Appl Polym Sci 2001 1998, 67.

3. Yeo, J. H.; Lee, C. H.; Park, C.-S.; Lee, K.-J.; Nam, J.-D..; Kim, S. W. Adv Polym Technol 2001, 20, 191.
4. Lasagabaster, A.; Abad, M. J.; Barral, L.; Ares, A. Eur Polymer J 2006, 42, 3121.

5. Lagaron, J. M.; Gimenez, E.; Saura, J. J.; Gavara, R. Polymer $2001,42,7381$.

6. Lagaron, J. M.; Gimenez, E.; Altava, B.; Del-Valle, V.; Gavara, R. Macromol Symp 2003, 198, 473.

7. Lagarón, J. M.; Giménez, E.; Gavara, R.; Saura, J. J. Polymer 2001, 42, 9531.

8. Lopez Rubio, A.; Hernandez-Muñoz, P.; Catala, R.; Gavara, R.; Lagaron, J. M. Food Addit Contam 2005, 22, 988.

9. Tsai, B. C.; Wachtel, J. A. In Barrier Polymers and Structures; Koros, W. J., Ed.; American Chemical Society: Washington, DC, 1990; p 192.

10. Lopez-Rubio, A.; Lagaron, J. M.; Gimenez, E.; Cava, D.; Hernandez-Muñoz, P.; Yamamoto, T.; Gavara, R. Macromolecules 2003, 36, 9467.

11. Lopez-Rubio, A.; Gavara, R.; Lagaron, J. M. J Appl Polym Sci 2006, 102, 1516.

12. Steele, D. Theory of Vibrational Spectroscopy; W.B. Saunders: London, 1971; p 134.

13. Hernandez, R. J.; Giacin, J. R.; Grulke, E. A. J Membr Sci 1992, 65, 187.

14. Lagaron, J. M.; Gimenez, E.; Catala, R.; Gavara, R. Macromol Chem Phys 2003, 204, 704 . 